\title{
FIBRONECTIN-LIKE IMMUNOREACTIVE STRUCTURES IN EMBRYONIC RAT BRAIN
}

\author{
Hiroshi Kimura, Ikuo Tohyama, Kounosuke Mizutani \\ and Junzo Ochi
}

Department of Anatomy, Shiga University of Medical Science, Otsu 520-21

\begin{abstract}
Fibronectin immunocytochemistry applied in trypsin-treated sections of embryonic rat brain revealed several positive structures. These include, in 18-dayembryo for example, somata of neuroblasts, ependymal cells, developing nerve terminals, meninges and blood vessels. Without trypsin treatment no positive immunoreactivity was seen in neural elements, though weak staining was observed only in meninges and capillaries. The neuroblasts possessing intracytoplasmic immunoreactivity for fibronectin were distributed in particular brain regions such as the cortical plate of the cerebrum and the primordium of the cerebellar nuclei. The intensity of immunostaining in neuronal and vascular structures gradually decreased or diminished at around perinatal periods. The results suggested that fibronectin may have important roles in neuronal migration or maturation. Moreover, fine positive processes ramified from capillaries suggested the role of fibronectin in neovascularization.
\end{abstract}

Fibronectin is a large glycoprotein found primarily in or in association with various types of connective tissue including blood, lymph, basement membranes, granulation tissue, amorphous elements of the intercellular matrix, and loose embryonic mesenchyme $(13,36,42)$. Fibronectin is synthesized by and secreted from a wide variety of cell types $(2,9,14,15,34,38)$. Amongst various functional activities of this glycoprotein, the property of binding to the cell surface is particularly interesting for the study of morphogenesis and cell differentiation of the brain. In fact, fibronectin is reported to be detected in glial cells in vitro including primary rat brain culture (19) and certain cell lines $(16,35)$, to stimulate some neuronal cell proliferation $(5,6)$, to guide the migration of neural crest cells (8), and to promote the outgrowth of neurites of embryonic chicken neural retina (1) or of human fetal sensory ganglia (4).

It may be worthy to note that laminin, an adhesive glycoprotein similar to fibronectin, has a specific role in neurite outgrowth and survival of neurons $(17,21$, 28). In addition, laminin has been demonstrated to be expressed in vivo after brain injury (18). More recently, a similar finding with fibronectin has been obtained in our study using a sensitive immunocytochemical method (23). What should be emphasized in that study is that not only glial cells but also neurons exhibited intracytoplasmic fibronectin-like immunoreactivity in the adult rat brain following cortical injury, while no immunostaining was seen in the neual component of intact rat brain. Immunoelectron microscopic examination further confirmed that fibronectin-like im-

Please send correspondence to: Hiroshi Kimura, M.D. 
munoreactivity was localized in axons and nerve terminals of corticofugal pathways.

These results led us to suggest that intraneuronal fibronectin has an important role in the regeneration processes of neurons, as seen in healing of skin wounds $(7,10$, 22). It is well known that some aspects of neuronal regeneration is akin to processes of brain development. It is expected, therefore, that fibronectin may be expressed in brain cells of embryonic rats. So far, however, no fibronectin has been demonstrated in embryonic rat brain. Here we applied a successful cytochemical technique to visualize fibronectin immunoreactivity in embryonic rat brain sections, and the results obtained mostly in 18-day embryos are presented in detail.

\section{MATERIALS AND METHODS}

Tissue Preparation Wistar rat strain was used. Newborn of fetuses on embryonic days $12,14,16$, or 18 were obtained. The day of conception was regarded as embryonic day zero. The crown-rump length (CR) of the embryos was measured, and CR 6 , 11,14 and $22 \mathrm{~mm}$ corresponded to the four gestational ages noted above, respectively. Embryos were removed from the uterus. Brains were rapidly fixed either by immersing for $20 \mathrm{~min}$ in a fixative or, for embryos older than day 16, by perfusing transcardially with the ice-cold fixative. The fixative consisted of $4 \%$ paraformaldehyde, $0.5 \%$ glutaraldehyde and $0.2 \%$ picric acid buffered with $0.1 \mathrm{M}$ sodium phosphate. The brain tissues were postfixed by immersing for 2 days in an ice-cold solution containing $4 \%$ paraformaldehyde and $0.2 \%$ picric acid in $0.1 \mathrm{M}$ phosphate buffer. The specimen was replaced and stored for 2 days in $0.1 \mathrm{M}$ phosphate buffer containing $15 \%$ sucrose at $4^{\circ} \mathrm{C}$. During storage the color of the specimens by yellow picrate turned faintly after several changes of the sucrose solution. After this cryoprotection step, the brain blocks were quickly frozen with dry-ice which was freshly prepared by ejecting liquid carbon dioxide from a needle valve. Serial sagittal sections of $30 \mu \mathrm{m}$ thick were cut in a cryostat and were stored for at least 4 days in phosphate buffered saline containing $0.3 \%$ Triton X-100 (PBST; $0.9 \% \mathrm{NaCl}$ in $0.1 \mathrm{M}$ sodium phosphate buffer, $\mathrm{pH} 7.4$ ). These sections were treated with either dimethylsulfoxide (DMSO) or trypsin shortly before processing for immunocytochemical staining procedures. For the DMSO treatment sections were immersed for 10-120 min in ice-cold $0.1 \mathrm{M}$ phosphate buffer containing $0.1-0.5 \% \mathrm{DMSO}$. For the trypsin digestion, sections were incubated for $30 \mathrm{~min}$ in $0.1 \mathrm{M}$ phosphate buffered saline containing $0.5 \mathrm{mM} \mathrm{CaCl}_{2}$ and $0.02 \%$ trypsin (w/v, Sigma, type III-S) at $37^{\circ} \mathrm{C}$. Immunocytochemistry The sections were incubated for 2 days with antiserum against fibronectin (diluted $1: 10,000$ ) at $4^{\circ} \mathrm{C}$, for $2 \mathrm{hr}$ with biotinylated anti-rabbit IgG (Vector Lab., diluted 1:800) at ambient temperature, and for $2 \mathrm{hr}$ with avidin-biotin-peroxidase complex (ABC; Vector Lab., Elite, diluted 1:2,000) at ambient temperature. They were colorized by reacting for 15 min with $0.05 \mathrm{M}$ Tris- $\mathrm{HCl}$ buffer $(\mathrm{pH} 7.5)$ containing $0.02 \% 3,3$ '-diaminobenzidine, $0.005 \% \mathrm{H}_{2} \mathrm{O}_{2}$ and $0.3 \%$ nickel ammonium sulfate. Dilution of the antibodies and the $\mathrm{ABC}$ complex as well as washing sections were done with PBST. Sections were always treated in a free-floating state throughout the whole staining procedure. The antisera against fibronectin was raised in rabbits by immunizing with fibronectin purified from canine plasma. Details of the antibody production as well as its immunochemical specificity have been published previously (23). For an immunohistochemical control, antifibronectin serum at work- 
ing dilution, which had been preincubated for 3 days with $1 \mu \mathrm{M}$ purified canine plasma fibronectin, was used as primary antibody. The stained sections were mounted on gelatin-coated glass slides. Some sections were counterstained with eosin for histological examination of brain structures.

\section{RESULTS}

In sections without DMSO or trypsin treatment, no immunoreactivity for fibronectin was found in neural structures irrespective of age of embryos (Fig. 1a). Meninges and blood vessels were, however, stained weakly in embryos older than day 16. The positive reaction in vessels was localized in vascular walls having relatively a large diameter. Such faintly stained blood vessels were distributed irregularly in some brain regions and primitive choroid plexus. A noticeable enhancement in staining intensity of these structures was observed in sections pretreated for 30 min with $0.5 \%$ DMSO (Fig. 1b), though no neural element was stained positively. In all ages studied, the meninges attached to the brain surface were more intensely stained than blood vessels. The meningeal staining, composed of both dura mater and leptomenix, was so intense and diffuse that the detail of the structure was not clearly visible. Stained blood vessels, varying in intensity and size, were found throughout various brain regions. In the absorption control study, positive staining was no more observed in sections treated with or without DMSO.

In trypsin-digested sections obtained from the 14-day-embryo onwards, distinct immunoreaction was revealed in some embryonic brain cells as well as blood vessels and meninges. Although positive neural cells appeared to be located in certain areas of the brain, it was often difficult to ascertain whether the positive brain cells were neurons or glial cells. This was particularly true in the case of embryos under 16 days; because the cells possessed immature shape of somata with few processes, and because extremely fragile sections were difficult to handle in order to permit systematic and detailed study of such positive brain cells. Fortunately, however, sections prepared from animals over embryonic day 18 were sufficiently strong against trypsin digestion for keeping the overall brain structure. Descriptions below therefore are mainly focused on observations in trypsinized sections from 14-day embryos.

Figure 2 illustrates a typical example of fibronectin-like immunoreactivity seen in the neocortex of CR length $22 \mathrm{~mm}$ embryo at the 18 th day of gestation. It was clearly observed that a significant number of positive cell bodies were localized in the cortical plate, while other layers contained few positive cells. Blood vessels and meninges were intensely stained in a similar way seen in DMSO-treated sections (Fig. 1b). With a higher magnification, numerous positive dotty puncta, probably developing nerve terminals, were deposited in the superficial part of the marginal zone of the cortex (Fig. 3a). In the cortical plate (Fig. 3b), the intense positive cells were mostly recognizable to be neuroblasts or neurons. They had oval or fusiform somata with a few dendritic processes and were clustered horizontally in a deep layer or vertically in a middle layer of the cortical plate. Figure $3 c$ shows the subventricular zone which contains many weakly stained cells, and beneath the zone there is the ventricular matrix cell zone. The latter zone was stained somewhat intensely, but the reaction products were diffusely distributed in the ependymal cell layer suggesting the occur- 


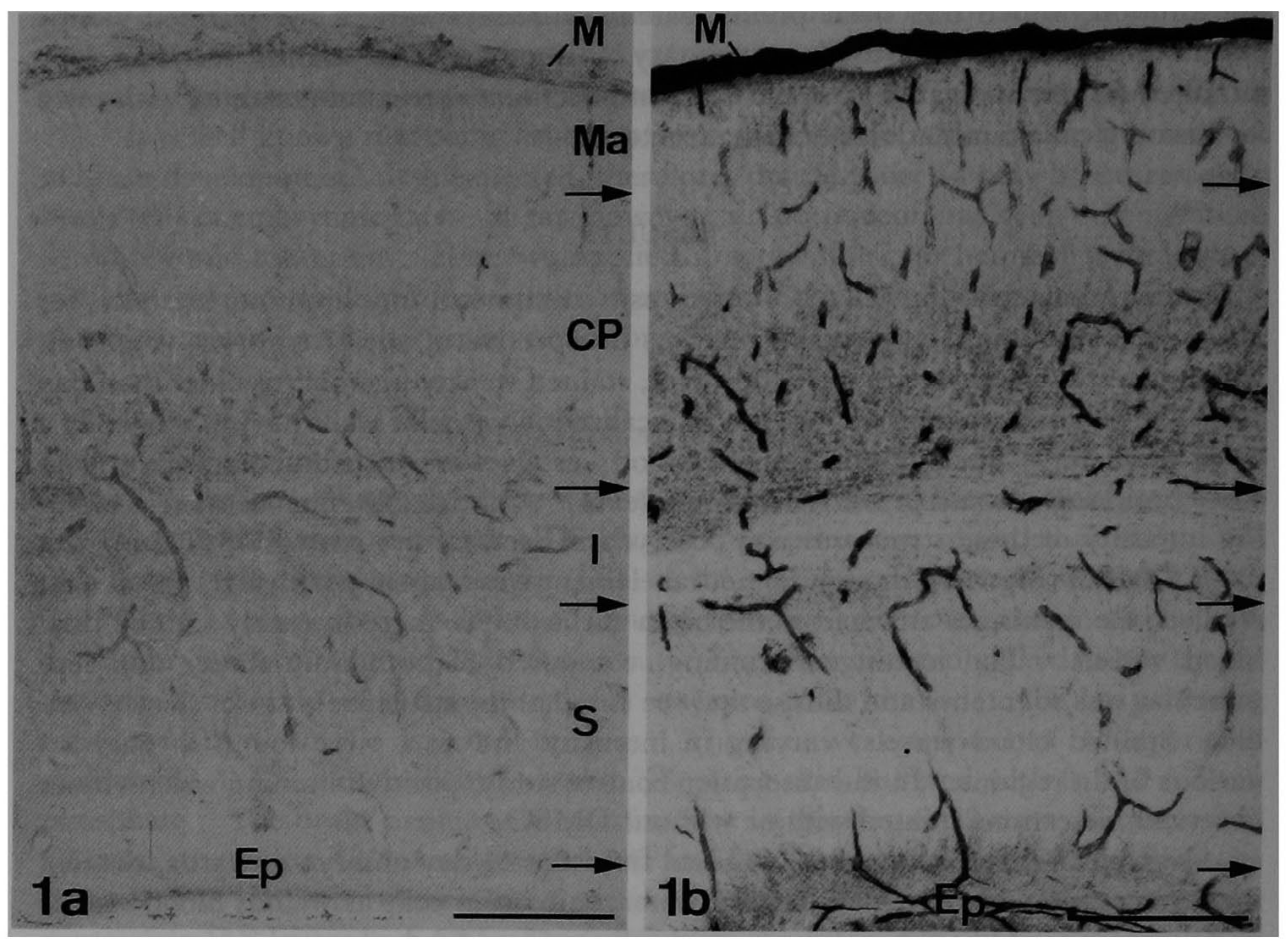

Fig. 1. Fibronectin immunocytochemistry in the rat cerebral cortex of 18-day embryo. Prior to immunostaining, the brain sections were treated with or without dimethylsulfoxide (DMSO). A section with no treatment (1a) shows faint staining only in a few capillaries which distribute in the cortical plate (CP), intermediate zone (I) and an upper part of the subventricular zone (S). In the meninges (M) a few dotty structures are also stained weakly. In contrast, a section with DMSO treatment $(1 \mathrm{~b})$ is stained intensely in the meninges as well as in capillaries which are seen throughout the entire cortical layers including the marginal (Ma) and ependymal (Ep) zones. Although a weak and diffuse staining is seen in the neuropil of CP, no cellular feature is recognizable. Bars $=200 \mu \mathrm{m}$.

FIg. 2. A section, treated with trypsin otherwise similar to that used in Fig. 1, demonstrates positive stainings in neuronal structures as well as the meninges and capillaries. See Fig. 1 for abbreviations. Note that strongly immunoreactive cell somata are clearly observed in $\mathrm{CP}$, and a moderate staining also occurs in $\mathrm{S}$ zone. Bar $=100 \mu \mathrm{m}$.

Fig. 3. A higher power magnification of three portions of Fig. 2. 3a: The marginal zone. Large arrows indicate immunoreactive blood vessels dereived from intensely positive meninges. Small arrows indicate terminal-like tiny puncta seen in a superficial layer just beneath the meninges. $3 b$ : The cortical plate. Large arrows indicate relatively large capillaries showing a high immunoreactivity. Small arrows exemplify neuroblasts possessing intracytoplasmic fibronectin-like immunoreactivity. In many occasions, nuclei of the positive neuroblasts are devoid of staining, and in a few cases uni- or bipolar processes are seen extending from the positive cells. 3c: the subventricular and ependymal (Ep) zones. Large arrows indicate intense and relatively large capillaries. A small arrow shows a fine fiber-like structure branching out of a positive capillary and connecting to another capillary. Bars $=50 \mu \mathrm{m}$. 


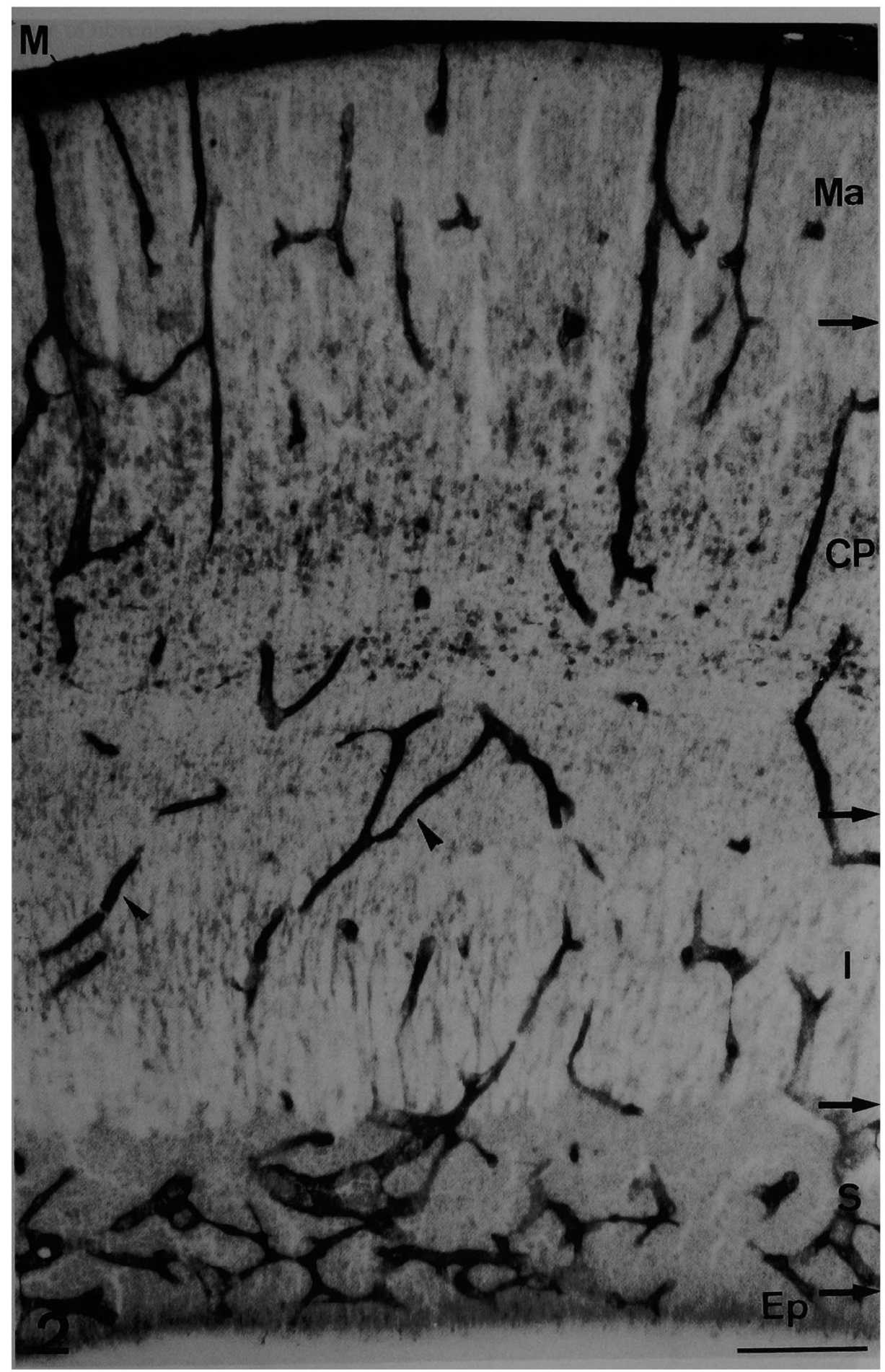


Kimura et al.

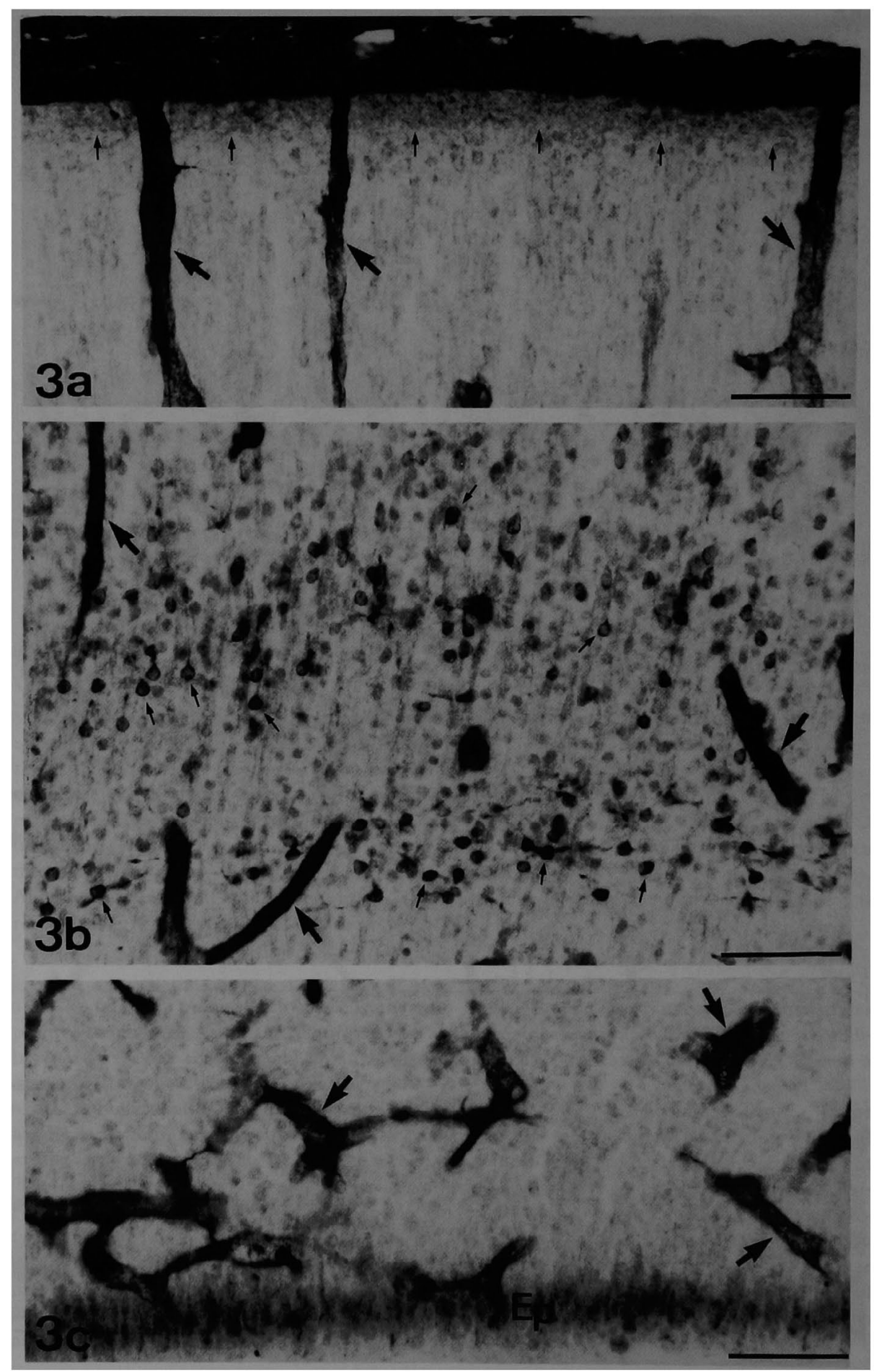


rence of fibronectin in the extracellular matrix. In the wall of the third ventricle (Fig. $4 a)$, however, ependymal cells occasionally showed intracytoplasmic immunoreactivity. Absorption control staining for trypsinized neighboring sections demonstrated no immunoreactivity in any structure including the ependymal cell layer (Fig. 4b).

The olfactory bulb displayed a particular laminar pattern of positive staining for fibronectin (Fig. 5a). The primordium of the anterior olfactory nucleus as well as developing mitral cell layer and external plexiform layer were stained weakly, while the border between the mitral cell layer and internal plexiform layer showed very high staining intensity. Even with a higher magnification (Fig. 5b), however, no obvious structure was determined to possess the intense immunoreaction products. These products seemed to be localized within fine processes of neural cells or in intercellular spaces. A similar staining pattern was observed in the border between the mitral cell layer and the external plexiform layer, though the the intensity of staining was generally kept in a moderate degree.

In the hindbrain, the cerebellum and the pontine tegmentum was noticed by the

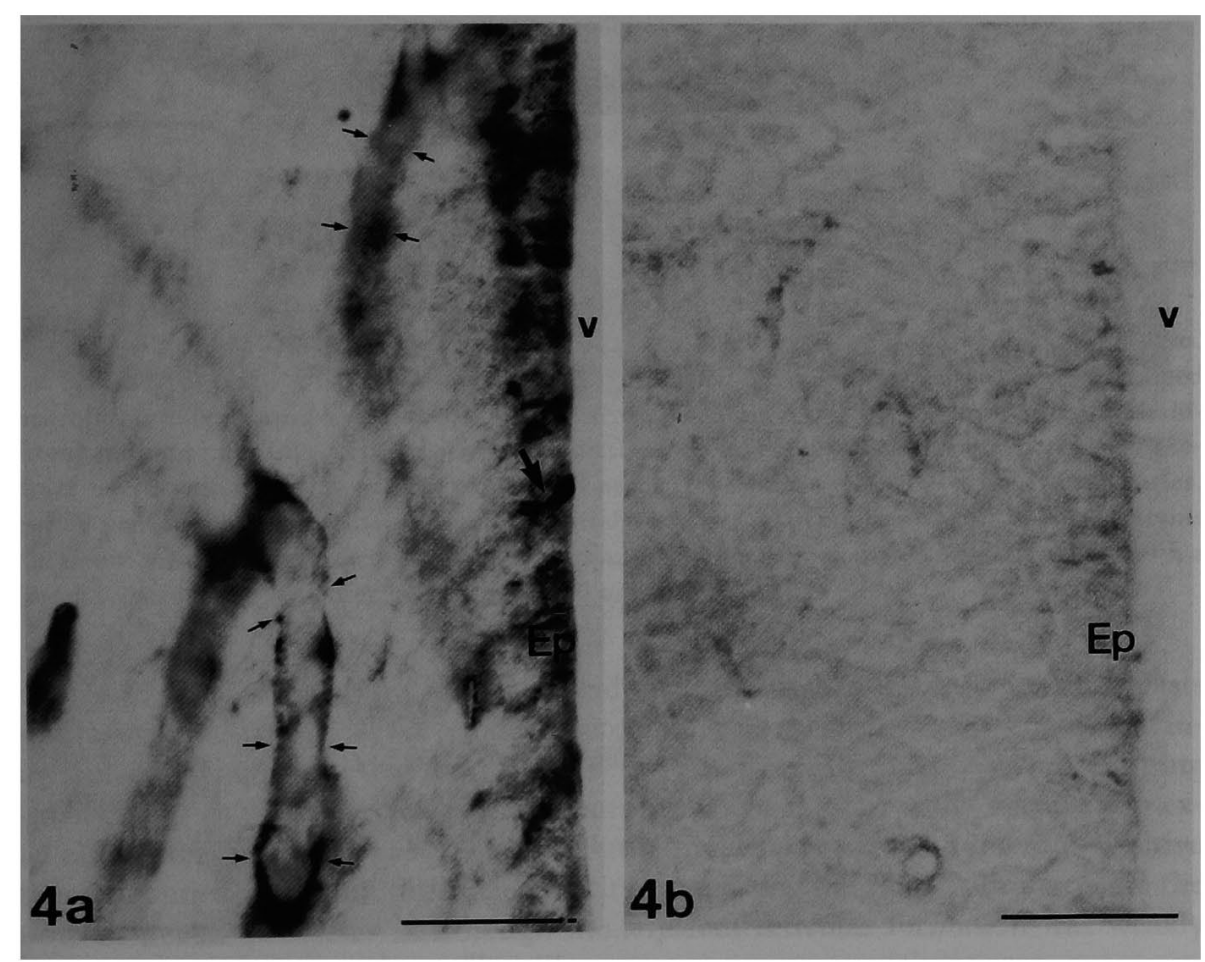

Fig. 4. The third ventricular wall in the hypothalamus of 18-day embryo. 4a: The ependymal cell zone (Ep) is stained somewhat diffusely and generally with a moderate density. Occasionally, a neuromatrix cell (large arrow) is clearly visible contacting with the third ventricle (v). In the hypothalamic region, little immunoreactive structure can be observed except for small capillaries whose outer surfaces are indicated by small arrows. 4b: An adjacent section to Fig. 4a, which has been stained with antiserum preabsorbed with excess amounts of fibronectin, shows no immunoreactivity. Bars $=50 \mu \mathrm{m}$. 

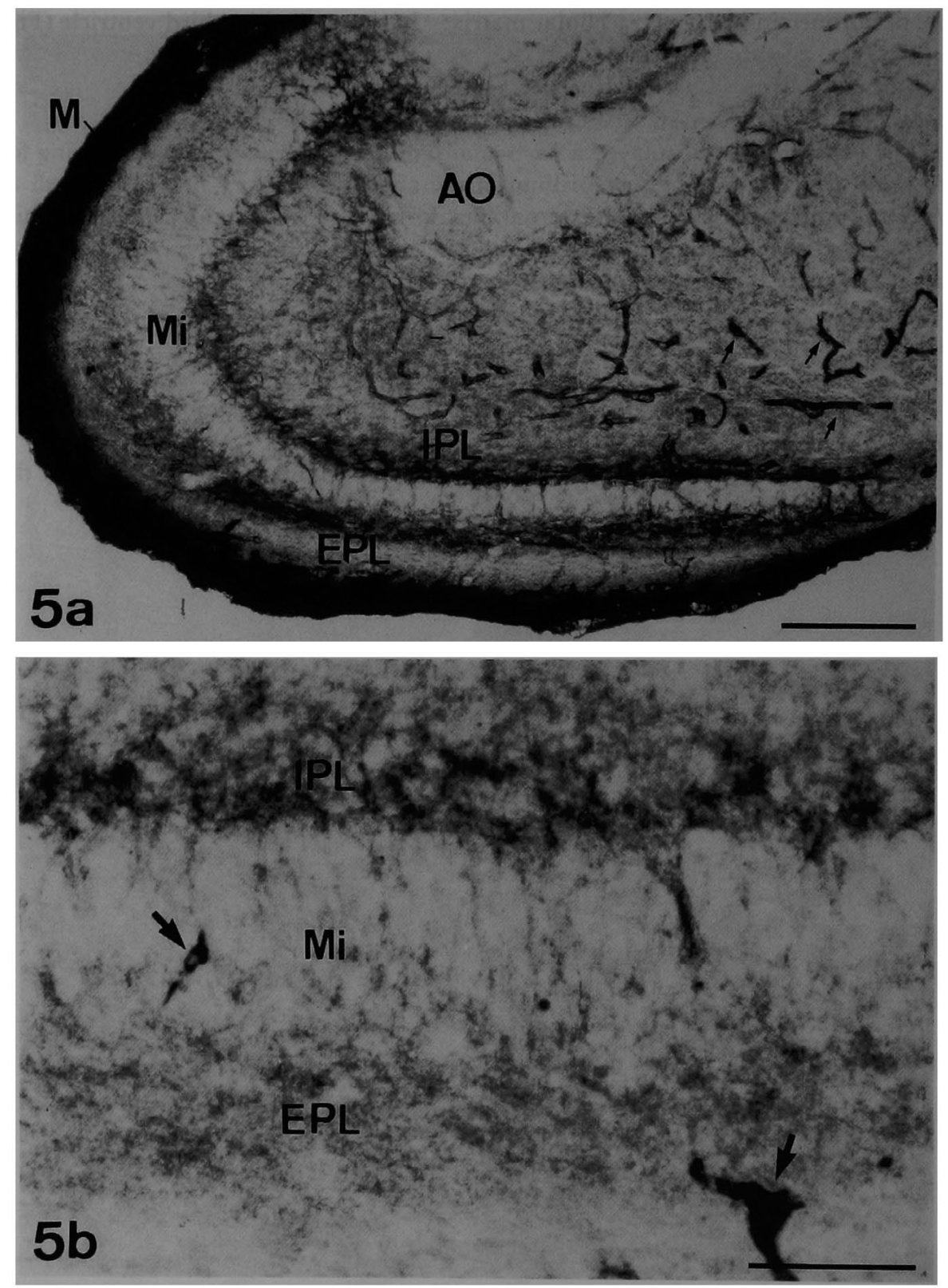

Fig. 5. Fibronectin immunocytochemistry in the olfactory bulb of 18-day embryo. 5a: Besides the intense positive meninges $(\mathrm{M})$ a specific pattern of immunoreactivity is seen such as a faint staining in the anterior olfactory nucleus ( $\mathrm{AO}$ ) and mitral cell layer $(\mathrm{Mi})$, and a moderate staining in the internal plexiform layer (IPL) and external plexiform layer (EPL). Small arrows indicate positive capillaries. Bar $=200 \mu \mathrm{m}$. 5b: A higher power magnification of a region around Mi. Note intense puncta deposited in IPL adjacent to Mi. A similar but less intense staining is seen in EPL neighboring to $\mathrm{Mi}$. Large arrows indicate positive capillaries. $\mathrm{Bar}=50 \mu \mathrm{m}$. 


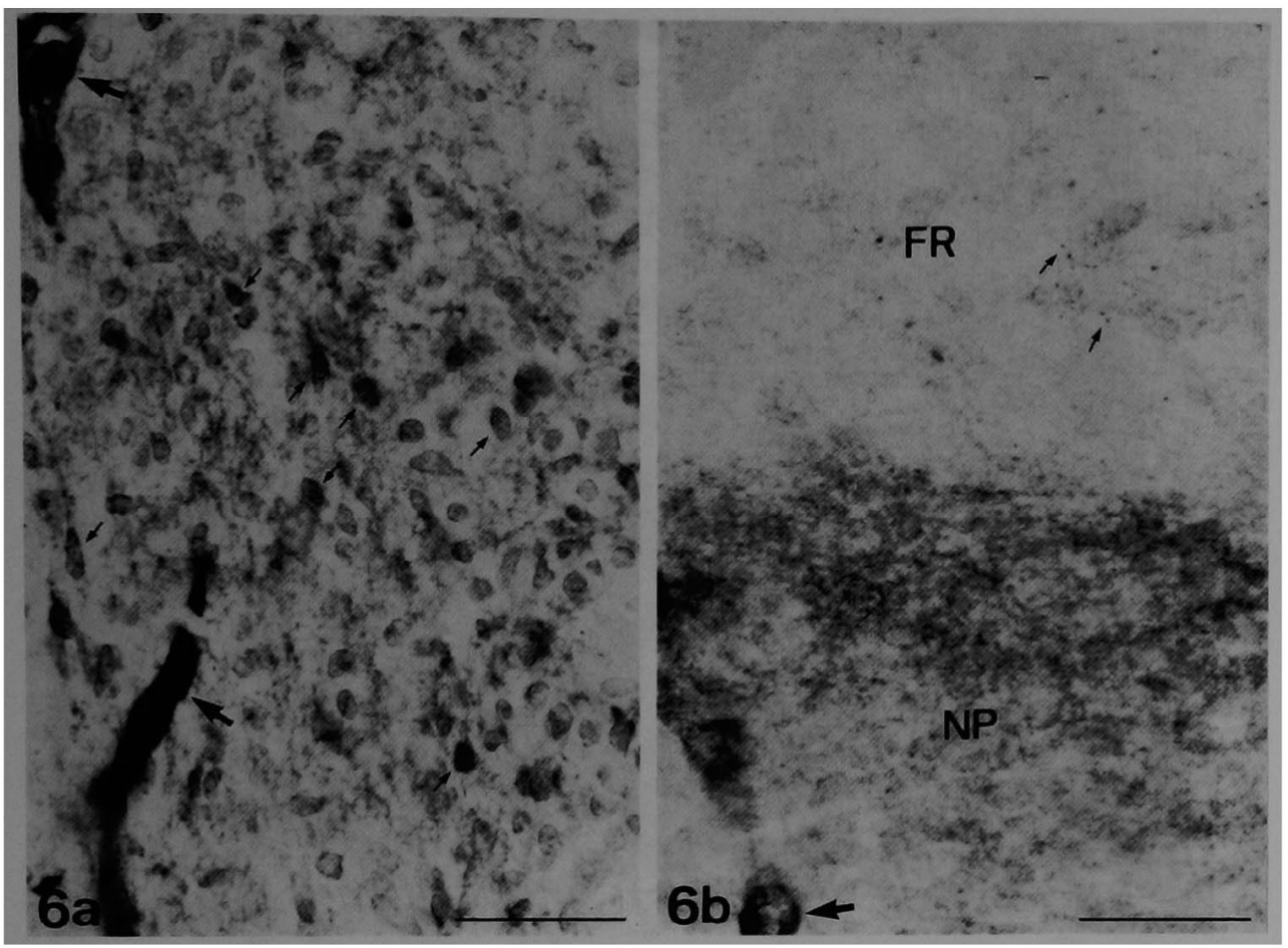

Fig. 6. Examples of fibronection positive neuronal structures in the hindbrain of 18-day embryo. 6a: A cluster of positive neuroblasts found in a core region of cerebellar buds. Variously shaped neuroblasts (a few examples indicated by small arrows) contain positive immunoreactivity with different staining intensity. The positive neuroblasts, some of which possess granular reaction products, are devoid of staining in their nuclei. Large arrows indicate intensely positive capillaries. 6b: A ventral portion of the pons in a sagittal section. The pontine nucleus (NP) contains numerous terminal-like puncta, while the reticular formation (FR) dorsal to NP is deposited with a few dotty structures (small arrows). Bars $=50 \mu \mathrm{m}$.

presence of positive staining in non-vascular elements. In a mass of neuroblasts situated in a deep part of developing cerebellum many cell bodies contained intracytoplasmic immunoreactivity. These cells varied greatly both in size and staining intensity (Fig. 6a). Large and intense neuroblasts were occasionally observed to extend a few dendritic processes. In the pontine nucleus (Fig. 6b) numerous positive dots were condensed witnin the nuclear entity, suggesting the role of fibronectin in the grwoth of neural processes. Although the adjacent reticular formation was weakly stained in general, fine varicose fiber-like structures were sometimes seen.

The pineal body was conspicuous because of a higher staining intensity than other brain regions (Fig. 7). The strong immunoreactivity was mainly found in large blood vessels which invaded from the meninges into the pineal parenchyma (Fig. 7a). There was little vascular staining in the stalk of the body around the pineal recess. In the parenchyma (Fig. 7b), a moderate intensity of staining was seen as thin and short fibrous structures. Such fibrous strands, scattering irregularly throughout the pineal 

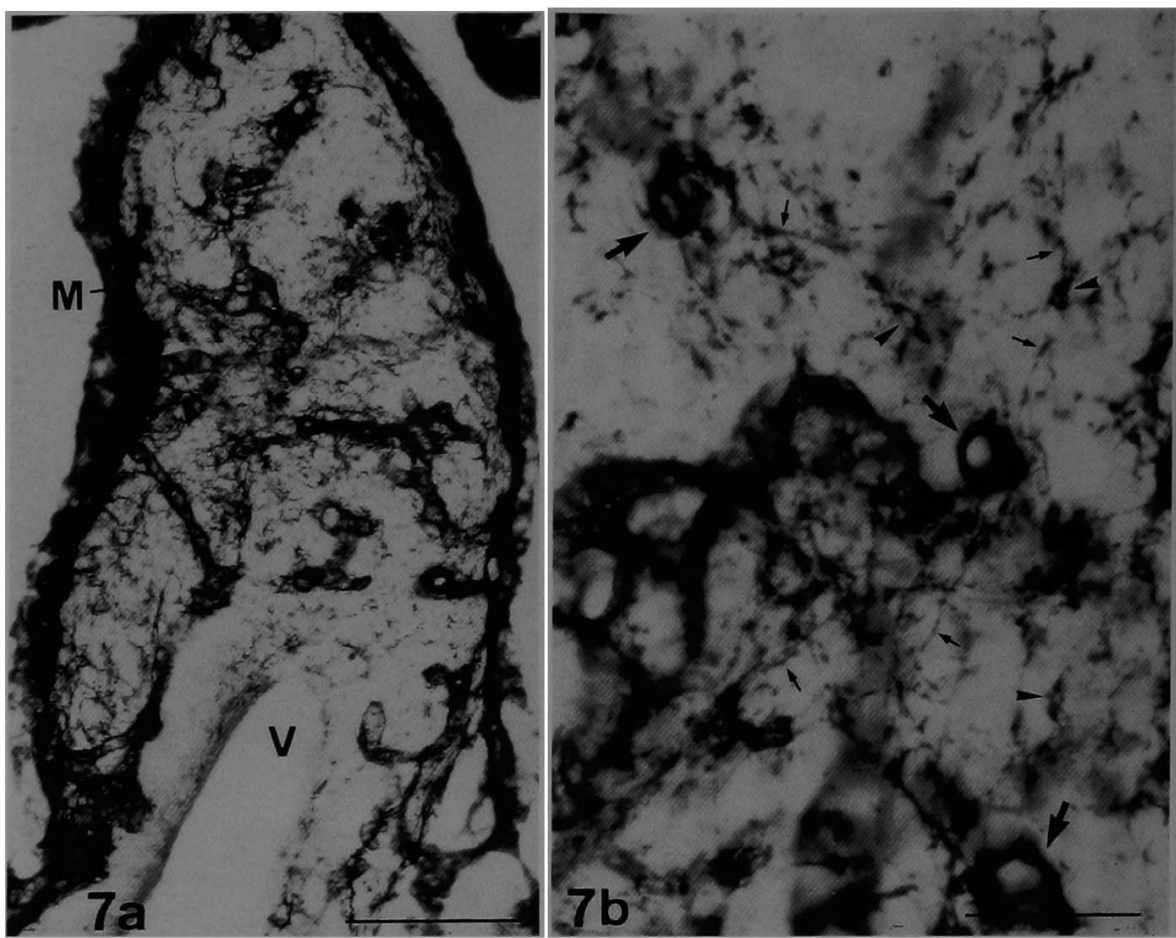

FIG. 7. The pineal body of 18-day embryo. The staining intensity in this structure is the highest among other brain regions. 7a: From the heavily stained meninges (M) are extended many intense capillaries. These capillaries as well as other positive structures are distributed throughout the pineal body, except for a faint staining in the pineal stalk around the dorsal third ventricle (V). $\mathrm{Bar}=100 \mu \mathrm{m}$. 7b: A higher magnification of Fig. 7a. Large arrows indicate intense capillaries, and small arrows indicate fiber-like processes possibly extending from positive capillaries. In addition, there are a few positive cells (arrow heads) which are stained with a moderate intensity. $\mathrm{Bar}=50 \mu \mathrm{m}$

body, appeared mainly derived from large positive vessels and partly related to pinealocytes. In fact, a few pinealoblasts showed intracytoplasmic immunoreactivity.

There were many brain regions manifesting the association of fibronectin with blood vessels, particularly with neovascularization. In the thalamus (Fig. 8a) and hypothalamus (Fig. 8b), for example, numerous capillaries were stained intensely. These capillaries, mostly sized less than $10 \mu \mathrm{m}$ in diameter, were so frequently branched out in every direction that anastomoses were commonly seen. On some occasions, moreover, very fine branches were ramified from positive capillaries extending to the parenchyma or to other capillaries. Since the diameter of the fine branching processes was $0.5 \mu \mathrm{m}$ or less, it was not possible to know whether or not its finest tips possess tubular structure.

As the growth of rats, both the density and intensity of all the immunoreactive structures described above was increased gradually up to day 18 . In perinatal infants, however, positive immunoreactivity was rapidly retarded from neural cells 


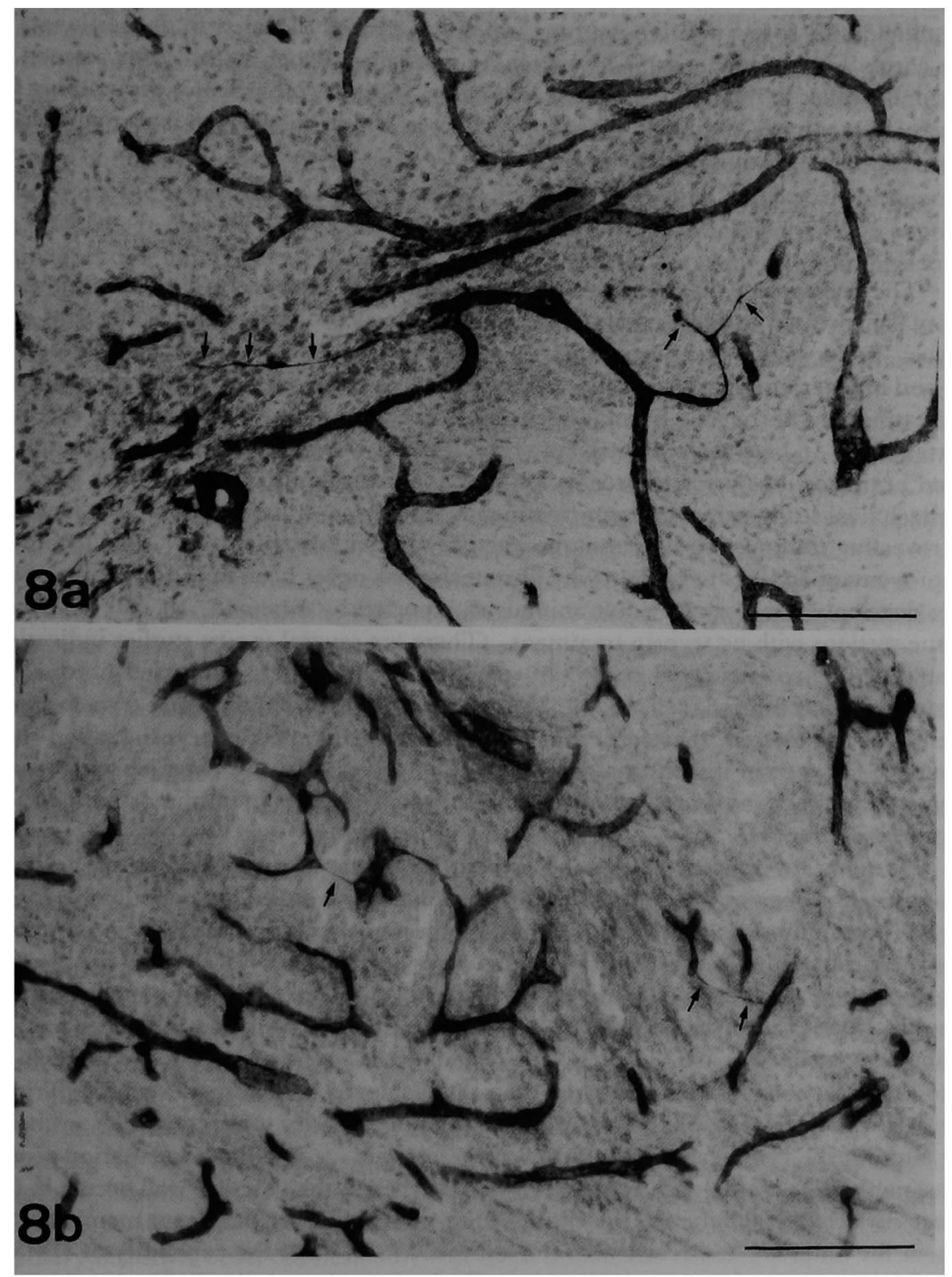

Fig. 8. Examples of fibronectin positive vascular elements in the thalamus (8a) and hypothalammus (8b). Small arrows indicate fine processes branched out of intense capillaries. Bars $=100 \mu \mathrm{m}$. 
(data not shown). In the neocortex of the 20th day embryo, for example, positive neuronal cells were scattered in the subcortical plate and much fewer numbers of positive cells were observed in the cortical plate. In a new born rat the cerebral cortex contained only a few positive neurons which were mostly located in deeper layers of the cortex (data not shown). The staining intensity of such neural cells seemed to become weaker in relation to brain development. On the other hand, the staining intensity of meninges and blood vessels were kept at a high level which is comparable to that seen in the 18th day embryo.

\section{DISCUSSION}

The present in vivo study demonstrates firstly that fibronectin-like immunoreactivity is expressed in some ependymal cells and developing neurons of intact rat embryos. Suppose that there is a similarity in certain mechanisms between developmental and regeneration processes, the present result accords well with our previous study indicating that fibronectin becomes detectable in regenerating neurons of the cerebral cortex (23). In both the previous and present study, we employed trypsin digestion of fixed brain sections prior to process for immunohistochemistry. As proven here by the technical study using different treatments of sections, trypsinization has a key role in revealing fibronection-like immunoreactivity in neural structures. This technical improvement appears to explain why fibronectin has never been found in brains of intact developing or lesioned adult animals as reported by others $(3,18,30)$ who used tissue sections with no trypsin treatment. There are several in vitro studies indicating that glial but not neuronal cells cultured from perinatal rats expressed fibronectin under certain conditions $(16,18,19,35)$. These studies also did not use trypsin treatment. It is expected, therefore, that a similar powerful effect of trypsinization may visualize fibronectin in neuronal cells of in vitro studies. In our experience, trypsintreated brain sections sometimes give better and stable results in immunostaining such as for serotonin (11).

Reasons for the success of the trypsin treatment are not known at present. However, to consider the underlying mechanisms may be valuable not only for an interest in immunostaining techniques but for the understanding of the true nature of fibronectin-like immunoreactivity. In this regard, the efficacy of DMSO for disclosing immunopositive vascular walls gives an important clue. Based on our empirical results, DMSO used in immunocytochemistry is helpful in some instances for the following possible reasons. Firstly, the reagent may accelerate antibody penetration into tissue sections in a different way than that achieved by detergents such as Triton $\mathrm{X}-100$. Secondly, DMSO may make a tissue antigen easier to react with its antibody by altering the chemical conformation of the antigen.. The first explanation seems reasonable because the positive reaction we observed in the vascular wall occurs in the outer surface of capillaries or the extracellular matrix like the basement membrane to whcih the antibody can reach with the aid of DMSO. But the capability of DMSO may be insufficient for penetrating the antibody through nerual cell membranes, and no intracytoplasmic immunostaining is obtained. The second possibility is much more interesting and should be discussed together with the present results obtained by the use of trypsin digestion. It has been well established that there are two general forms of fibronectin differing in solubility and tissue localization (13). Soluble 
fibronection is mainly found in plasma and lymph, while insoluble fibronectin is distributed in tissue or cellular components. Plasma and tissue fibronectins are very similar (molecular weight of both forms; about $450-\mathrm{kDa}$ ) but not identical in chemical conformation $(27,39,40)$. The targets of trypsin digestion are predominantly focused on the peptide linkage between arginine and phenylalanine, and rat tissue fibronectin contains three occasions of the amino acid sequence $(24,25,31,32)$. Smaller peptide fragments of intracellular fibronectin are thus expected to be produced in fixed brain sections by proteolytic reaction of trypsin and of DMSO as well. Under such conditions the antiserum we used might be able to recognize some of these peptide fragments. It may be noteworthy, in addition, that trypsin-digested 75-kDa peptide fragments of human plasma fibronection possess particularly potent cell-binding activity $(12,41)$.

So far no information is available concerning the orgins of embryonic brain fibronectin. This may be because of a difficulty in detection of fibronectin whose immunoreactivity alters dramatically during perinatal periods as shown in this study. Another difficulty is in the fact that the concentration of the cellular type fibronectin found in plasma occupies only $1 \%$ of the plasma fibronectin which is predominantly secreted by hepatocytes $(34,38)$. Besides liver cells, however, fibronectin has been shown to be produced by various cell types including fibroblasts (9), macrophages (15), vascular endothelial cells (14), some types of lung cells (29), and glial cells (19) as well as other parenchymal cells of various organs. It is not surprising therefore that neuronal cells may synthesize fibronectin in some stages of development. Another possibility is that plasma fibronectin is taken up by embryonic neuronal cells. To answer this question the immunocytochemcial approach using a specific antibody to celllar fibronectin $(26,37)$ or in situ hybridization for mRNA should provide valuable informaton.

The role of neuronal fibronectin is yet unknown. However, as suggested by our previous study (23), fibronectin may act on neurite outgrowth by promoting cellmatrix interaction. In this context, it is important to note that the positive immunoreactivity gradually disappears after the completion of neuronal migration or maturation. It is also interesting to speculate neuronal fibronectin may have some role in synaptogenesis. The heavily stained dotty structures exemplified in the pontine nucleus and the cortical marginal layer appear to support the hypothesis. Fibronectin is a cell-to-cell adhesive protein, and synaptogenesis is regarded as one type of cell-to-cell adhesion. To examine this possibility more detailed ontogenetic study in much younger embryos should be carried out when the problem in trypsinization inherent to our technique is overcome.

Besides the positive immunoreactivity in neural elements, very intense staining occurring in blood vessels suggests the role of fibronectin in neovascularization. Vascular fibronectin, which is thought to derive mainly from plasma hepatocyte origin, deposits as an insoluble form in the basement membrane (29). Thus fiber-like capillary branches observed here may represent fibronectin deposited in the basement membrane which has a role of pilot in neovascularization. A similar role in neovascularization was obtained with laminin which appeared during early stages of brain transplant (33). 


\section{ACKNOWLEDGMENT}

This study was partly supported by the Grant-in-Aid from Ministry of Education, Science and Culture (No. 62221017; H.K.). A part of this original paper was presented in symposium of the Japan Society for Cytochemistry and histochemistry at Utsunomiya on October 28, 1988.

\section{REFERENCES}

1. Akers, R.M., Mosher, D.F. and Lilien, J.E.: Promotion of retinal neurite outgrowth by substratum-bound fibronectin. Dev. Biol. 86; 179-188, 1981.

2. Alitalo, K., Hovi, T. and Vaheri, A.: Fibronectin is produced by human macrophages. $J$. Exp. Med. 151; 602-613, 1980.

3. Alitalo, K., Kurikinen, M., Virtanen, I., Mellstrom, K. and Vaheri, A.: Deposition of basement membrane proteins in attachment and neurite formation of cultured murine c- 1300 neuroblastoma cells. J. Cell Biochem. 18; 25-36, 1982.

4. Baron-Van Evercooren, A., Kleinman, H.K., Ohno, S., Marangos, P., Schwartz, J.P. and Dubois-Dalcq, M.E.: Nerve growth factor, laminin and fibronectin promote neurite grwoth in human sensory ganglia cultures. J. Neurosci. Res. 8; 179-193, 1982.

5. Baron-Van Evercooren, A., Kleinman, H.K., Seppa, H.E.J., Rentier, B. and Dubois-Dalcq, M.E.: Fibronectin promotes rat Schwan cell growth and motility. J. Cell Biol. 93; 211-216, 1982.

6. Bottenstein, J.E. and Sato G.H.: Fibronectin and polyserine requirement for proliferation of neuroblastoma cells in defined medium. Exp. Cell Res. 129; 361-366, 1980.

7. Colvin, R.B.: Fibronectin in wound healing. In Fibronectin, ed. by D.F. Mosher, Academic Press, Inc., San Diego, 1989, p. 213-254.

8. Duband, J.-L., and Thiery, J.P.: Distribution of fibronectin in the early phase of avian cephalic neural crest cell migration. Dev. Biol. 93; 308-323, 1982.

9. Engvall, E. and Rouslahti, E.: Binding of soluble form of fibroblast surface protein, fibronectin, to collagen. Int. J. Cancer 20;1-5, 1977.

10. Grinnel, F.: Fibronectin and wound healing Am. J. Dermatopathol. 4; 185-188, 1982.

11. Hara, K., Tohyama, I., Kimura, H., Fukuda, H., Nakamura, S. and Kameyama, M.: Reversible serotoninergic neurotoxicity of $N$-methyl-4-phenyl-1, 2, 3, 6-tetrahydropyridine (MPTP) in mouse striatum studied by neurochemical and immunohistochemical approaches. Brain Res. 410; 371-374, 1987.

12. Hayashi, M. and Yamada, K.M.: Domain structure of the carboxyl terminal half of human plasma fibronectin. J. Biol. Chem. 258; 3332-3340, 1983.

13. Hynes, R.O. and Yamada, K.M.: Fibronectins, multifunctional modular glycoproteins. J. Cell Biol. 95; 369-377, 1982.

14. Jaffe, E.A. and Mosher, D.F.: Synthesis of fibronectin by cultured human endothelial cells. J. Exp. Med. 147; 1779-1791, 1978.

15. Johnsson, S., Rubin, K., Hook, M., Ahlgren, T. and Seljelid, R.: In vitro biosynthesis of cold insoluble globulin (fibronectin) by mouse. FEBS Lett. 105; 313-316, 1979.

16. Jones, T.R., Rouslahti, E., Schold, S.C. and Bigner, D.D.: Fibronectin and glial fibrillary acidic protein expression in normal human brain and anaplastic human gliomas. Cancer Res. 42; 168-177, 1982.

17. Liesi, P., Dahl, D. and Vaheri, A.: Neurons cultured from developing rat brain attach and spread preferentially to laminin. J. Neurosci. Res. 11; 241-252, 1984.

18. Liesi, P., Kaakkola, S., Dahl, D. and Vaheri, A.: Laminin is induced in astrocytes of adult brain by injury. EMBO J. 3; 683-686, 1984. 
19. Liesi, P., Kirkwood, T. and Vaheri, A.: Fibronectin is expressed by astrocytes cultured from embryonic and early postnatal rat brain. Exp. Cell Res. 163; 175-185, 1986.

20. McDonald, J.A.: Fibronectin in the lung. In Fibronectin, ed. by D.F. Mosher, Academic Press, Inc., San Diego, 1989, p. 363-393.

21. Manthorpe, M., Engvall, E., Rouslahtu, E., Longo, F., Davis, G. and Varon, S.: Laminin promotes neuritic regeneration from cultured peripheral and central neurons. J. Cell Biol. 97; 1882-1890, 1983.

22. Matsuda, M.: Fibronectin and its related substances-their roles in tissue repair. Connect. Tissue 13; 177-184, 1982.

23. Mizutani, K. and Kimura H.: Immunohistochemical studies of fibronectin immunoreactive structures demonstrated in response to brain injury of adult rats. Acta histochem. cytochem. 20; 87-99, 1987.

24. Odermatt, E. Tamkun, J.W. and Hynes, R.O.: Releasing modulator structure of the fibronectin gene relationship to protein structure and subunit variation. Proc. Natl. Acd. Sci. U.S.A. 82; 6571-6575, 1985.

25. Patel, R.S., Odermatt, E., Schwarzbauer, J.E. and Hynes, R.O.: Organization of fibronectin gene provides evidence for exon shuffling during evolution. $E M B O J .6 ; 2565-2572,1987$.

26. Peters, J.H., Ginsberg, M.H., Bohl B.P., Sklar, L.A. and Cochrane, C.G.: Intravascular release of intact cellular fibronectin during oxidant-induced injury of the in vitro perfused rat lung. J. Clin. Invest. 78; 1596-1603, 1986.

27. Petersen, T.E., Skorstengaard, K. and Vibe-Pedersen, K.: Primary structure of fibronectin. In Fibronectin, ed. by D.F. Mosher, Academic Press, Inc., San Diego, 1989, p. 1-24.

28. Rogers, S., Letourneau, P., Palm, S., McCarthy, J. and Furcht, L.: Neurite expansion by peripheral and central nervous system neurons in response to substratum-bound fibronectin and laminin. Dev. Biol. 98; 212-220, 1983.

29. Saba, T.M.: Kinetics of plasma fibronectin: relationship to phagocytic function and lung vascular integrity. In Fibronectin, ed. by D.F. Mosher, Academic Press, Inc., San Diego, 1989 , p. $395-439$.

30. Schachner, M., Schoonmaker, G. and Hynes, R.O.: Cellular and subcellular localization of LETS protein in the nervous system. Brain Res. 158; 149-158, 1978.

31. Schwarzbauer, J.E., Tamkun, J.W., Lemischka, I.R. and Hynes, R.O.: 3 fibronectin messenger RNA species arise by alternative splicing within the coding region. Cell $35 ; 421-$ $431,1983$.

32. Schwarzbauer, J.E., Patel, R.S., Fonda, D. and Hynes, R.O.: Multiple sites of alternative splicing of the rat fibronectin gene transcript. $E M B O J .6 ; 2573-2580,1987$.

33. Shigematsu, K., Kamo, H., Akiguchi, I., Kameyama, M. and Kimura, H.: Neovascularization of transplanted central nervous tissue suspensions: an immunohistochemical study with laminin. Neurosci. Lett. (in press)

34. Tamukun, J.W. and Hynes, R.O.: Plasma fibronectin is synthesized and secreted by hepatocytes. J. Biol. Chem. 258; 4641-4647, 1983.

35. Vaheri, A., Rouslahti, E., Westermark, B. and Ponten, J.: A common cell-type specific surface antigen in cultured human glial cells and fibroblasts: loss in malignant cells. J. Exp. Med. 143; 64-72, 1976.

36. Vartio, T. and Vaheri, A.: Fibronectin-chains of domains with diversified functions. Trends Biochem. Sci. 8; 442-444, 1983.

37. Vartio, T., Laitinen, L., Narvanen, O., Thornell, L.E. and Virtanen, I.: Differential expression of the ED sequence-containing form cellular fibronectin in embryonic and adult human tissues. J. Cell Sci. 88; 419-430, 1987.

38. Voss, B., Allam, S., Rauterberg, J., Ullrich, K., Gieselmann, V. and Von Figura, K.: Primary cultures of rat hepatocytes synthesize fibronectin. Biochem. Biophys. Res. Commun. 90; 1348-1354, 1979.

39. Yamada, K.M.: Fibronectin domains and receptors. In Fibronectin, ed. by D.F. Mosher, Academic Press, Inc., San Diego, 1989, p. 48-121. 
40. Yamada, K.M. and Kennedy, D.W.: Fibroblast cellular and plasma fibronectin are similar but not identical. J. Cell Biol. 80; 492-498, 1979.

41. Yamada, K.M. and Kennedy, D.W.: Dualistic nature of adhesive protein fibronectin and its biologically active peptide fragments can autoinhibit function. J. Cell Biol. 99; 29-36, 1984.

42. Yamada, K.M. and Olden, K.: Fibronectins-adhesive glycoproteins of cell surface and blood. Nature (London) 275; 179-184, 1978. 